\title{
Driving/Braking Force Distribution of Four Wheel Vehicle by Quadratic Programming with Constraints*
}

\author{
Yuichi IKEDA**, Ryo SUZUKI** and Yuichi CHIDA** \\ ** Shinshu University \\ 4-17-1 Wakasato, Nagano, Nagano 380-8553, Japan \\ E-mail: yikeda@shinshu-u.ac.jp
}

\begin{abstract}
This paper proposes a yaw rate tracking control method that distributes the driving/ braking force exerted on vehicles at the time of negotiating sharp turns and driving at high speeds. The proposed method employs quadratic programming to distribute the driving/braking force in order to equalize the tire load factor on all wheels and consider the limits of the driving/braking force. The yaw rate tracking performance can be improved even while driving at high speeds and negotiating sharp turns by setting limits for the driving/braking force, differential moment, etc. The effectiveness of our proposed method is proven by a numerical simulation.
\end{abstract}

Key words : Four Wheel Vehicle, Driving/Braking Force Distribution, Quadratic Programming, Constraints

\section{Introduction}

Electric vehicles have attracted considerable attention in recent years because of their potential advantages over vehicles having conventional internal combustion engines. Electric vehicles utilize electric motors instead of internal combustion engines, which have advantages such as a small environmental load because of the absence of exhaust gases and low noise. However, the most important advantage is their superior responsiveness and easy controllability.

Until now, the driver could only control the vehicle using the steering wheel and the accelerator/brake pedal. However, electric vehicles have the capability to be individually driven at each wheel independently by using in-wheel motors. The difference between driving/braking forces of these motors produces a yaw moment that can be utilized as a new control input. Direct yaw moment control (DYC) is an attitude control method that utilizes this yaw moment. It is realized by distributing the appropriate driving/braking force to each wheel. Employing DYC offers many advantages. For example, the yaw rate of the vehicle can be controlled with a high accuracy, which makes cornering smoother when driving along curves. Moreover, the vehicle remains stable even while driving along split- $\mu$ road surfaces, which leads to greater driving comfort and prevention of traffic accidents.

DYC has been proposed in various research studies. Some methods proposed for distribution include the weighted least mean squares method ${ }^{(1),(2)}$, methods based on the speed differential between each wheel ${ }^{(3)}$, and methods employing the minimax optimization method ${ }^{(4)}$. In addition, it is important to take into consideration the limitation of the motor output torque in DYC because the motor output saturates at the time that vehicle is driven at high speeds and used to negotiate sharp turns because of a large differential moment, i.e., a large driving/braking force is required at such instances. Consequently, the control performance cannot be sustained and the vehicle's overall performance may deteriorate. References (1) and (2) take action that the commanded torque from the driver is restricted and generation of driving/braking force differential moment is prioritized when the motor output torque exceeds the 
limiter. However, the torque in performance limitation cannot be outputted and thus the vehicle velocity cannot be accelerated and/or deaccelerated enough since the limitation of motor output torque is not considered theoretically. While method of Ref. (4) considers generation of the commanded value that each tire forces are not saturated, limitation of motor output torque is not considered. Methods that consider the torque limitations while distributing the driving/braking force have also been reported ${ }^{(5)-(7)}$. The methods proposed in Ref. (5) and Ref. (6) utilize a controller designed to track the yaw rate command and control the distribution of the driving/braking force by quadratic programming to match the inputs generated by the controller. However, the control performance depends entirely on the controller. Moreover, in the method proposed in Ref. (6), the controller is designed such that the lateral force on the tire is linear with respect to the sideslip angle when the vehicle speed is constant; however, the method does not sustain the performance when comparatively large changes in sideslip angle or speed occur. On the other hand, the method proposed in Ref. (7) uses multiparametric quadratic programming to distribute the driving/braking force. In this method, the computation load is small because the distributed driving/braking forces are calculated offline; however, applying this method is difficult when the computer memory mounted on the vehicle is limited. In addition, vehicle motion stability cannot be guaranteed if the vehicle deviates from the assumed preset range while driving.

Taking these problems into account, we have proposed a method for distributing the driving/braking force by quadratic programming, which takes into account the constraints of the driving/braking force limitation of each wheel, even when the driving tends to become unstable such as while driving at high speeds and negotiating sharp turns. Although methods using quadratic programming for distributing the driving/braking force have been proposed as described previously, sufficient driving/braking differential moment for turn of vehicles may not be generated because these methods only consider the constraints of the driving/braking force limitation. In proposed method, by adding the constraint that increases the driving/braking differential moment as much as possible under the constraints of the driving/braking force limitation, it prevents falling into this situation. Furthermore, we also propose a control system based on yaw moment compensation to prevent the deterioration of control performance when the limits are exceeded. The effectiveness of the proposed method is proven through a numerical simulation of the yaw rate tracking control using a nonlinear vehicle model that takes into account the changes in speed due to acceleration and deceleration.

\section{Vehicle Model}

The vehicle and wheel models are shown in Figs. 1 and 2, respectively, and the physical parameters of the vehicles are listed in Table 1. The physical parameters in Table 1 determined by reference to a commercial-type vehicle and References (1) and (2). Here, a four-wheel drive vehicle with internal in-wheel motors on each wheel is considered, and it can be steered only by the front steering. In Figs. 1 and $2, v_{x}[\mathrm{~m} / \mathrm{s}]$ is the vehicle velocity of longitudinal direction; $v_{y}[\mathrm{~m} / \mathrm{s}]$ is the vehicle velocity of lateral direction; $\gamma[\mathrm{rad} / \mathrm{s}]$ is the yaw rate around the center of mass of the vehicle; $\delta$ [rad] is the steering angle; $\beta_{i j}[\mathrm{rad}]$ are the sideslip angles at each wheel; $F_{x i j}[\mathrm{~N}]$ are the driving/braking forces on each wheel; $F_{y i j}[\mathrm{~N}]$ are the lateral forces on each wheel; $\lambda_{i j}[-]$ is the slip ratio of each wheel; $\omega_{i j}[\mathrm{rad} / \mathrm{s}]$ is the angular velocity of each wheel; and $\tau_{i j}[\mathrm{Nm}]$ is the driving/braking torque on each wheel ( $i \in\{f, r\}, j \in\{l, r\})$. The longitudinal and lateral motion of the vehicle's center of gravity, rotational motion around the yaw axis, and the wheel rotational motion are expressed by the following equations:

$$
\begin{aligned}
& m \dot{v}_{x}=m v_{y} \gamma+F_{x}, \\
& m \dot{v}_{y}=-m v_{x} \gamma+F_{y}, \\
& J \dot{\gamma}=N_{z}+N_{t}=M_{z},
\end{aligned}
$$



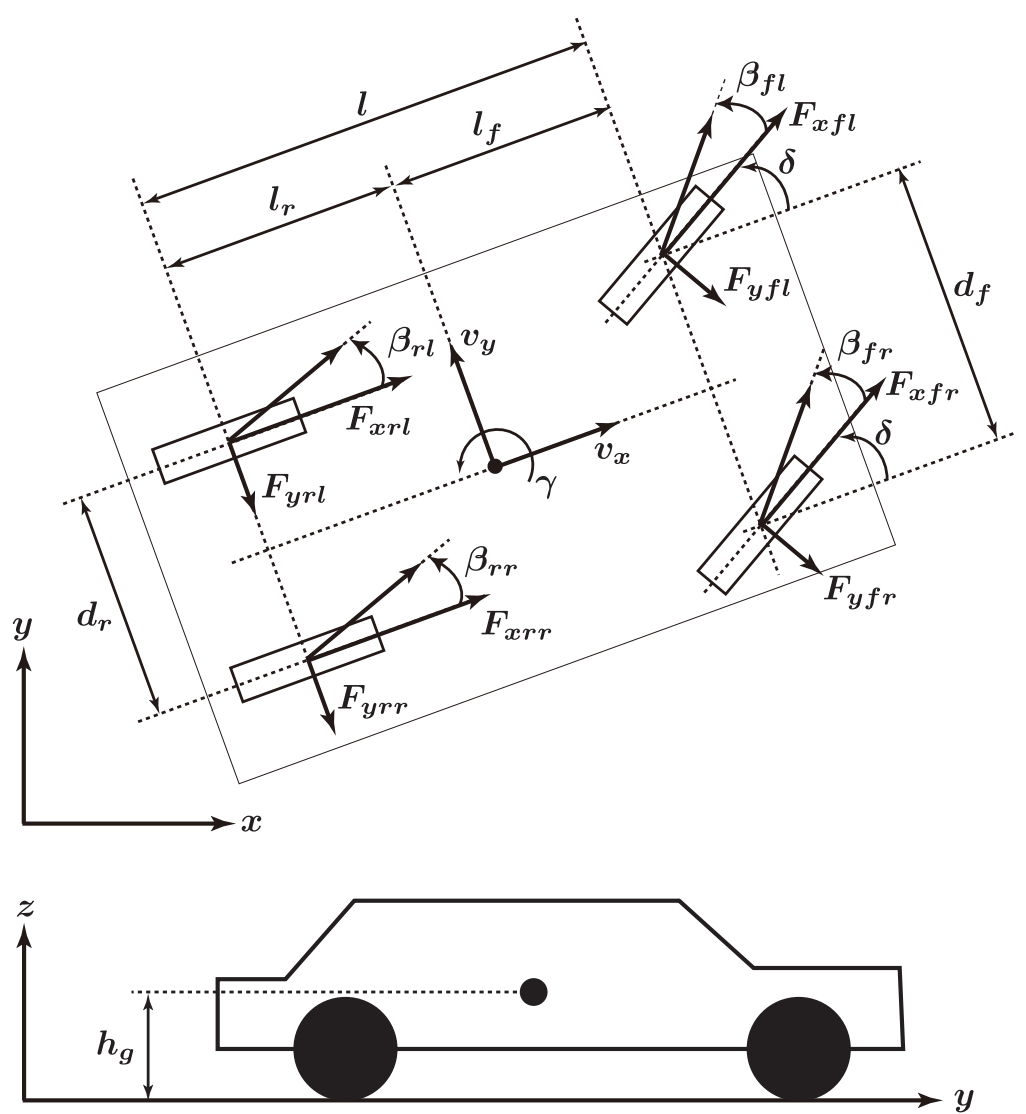

Fig. 1 Vehicle model

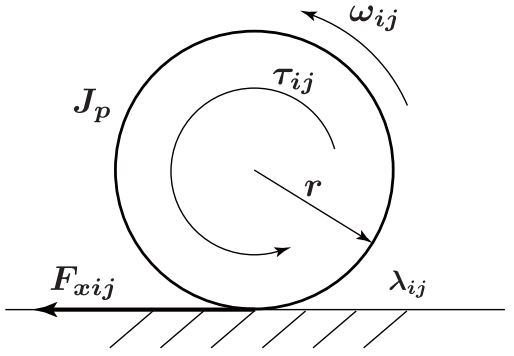

Fig. 2 Wheel model

$$
J_{p} \dot{\omega}_{i j}=\tau_{i j}-r F_{x i j},
$$

where $F_{x}$ and $F_{y}$ are the longitudinal and lateral force acting at the vehicle's center of gravity, respectively, $M_{z}$ is the yaw moment, $N_{z}$ is the driving/braking force differential moment, and $N_{t}$ is the lateral force moment. $F_{x}, F_{y}, N_{z}$, and $N_{t}$ derived from the driving/braking force $F_{x i j}$ and lateral force $F_{y i j}$ on each wheel are given by the following equations:

$$
\begin{aligned}
F_{x}= & \left(F_{x f l}+F_{x f r}\right) \cos \delta+F_{x r l}+F_{x r r}+\left(F_{y f l}+F_{y f r}\right) \sin \delta, \\
F_{y}= & \left(F_{x f l}+F_{x f r}\right) \sin \delta-\left(F_{y f l}+F_{y f r}\right) \cos \delta-\left(F_{y r l}+F_{y r r}\right), \\
N_{z}= & \left(l_{f} \sin \delta-\frac{d_{f}}{2} \cos \delta\right) F_{x f l}+\left(\frac{d_{f}}{2} \cos \delta+l_{f} \sin \delta\right) F_{x f r} \\
& -\frac{d_{r}}{2}\left(F_{x r l}-F_{x r r}\right),
\end{aligned}
$$


Table 1 Physical parameters of vehicle model

\begin{tabular}{|c|c|c|}
\hline$m[\mathrm{~kg}]$ & Vehicle mass & 1415 \\
\hline$m_{s}[\mathrm{~kg}]$ & Sprung mass & 1300 \\
\hline$m_{s f}[\mathrm{~kg}]$ & Front unsprung weight & 32.5 \\
\hline$m_{s r}[\mathrm{~kg}]$ & Rear unsprung weight & 25 \\
\hline$J\left[\mathrm{kgm}^{2}\right]$ & Inertia moment of vehicle & 2646 \\
\hline$l_{f}[\mathrm{~m}]$ & $\begin{array}{c}\text { Center of gravity-front } \\
\text { distance between wheels }\end{array}$ & 1.2 \\
\hline$l_{r}[\mathrm{~m}]$ & $\begin{array}{c}\text { Center of gravity-rear } \\
\text { distance between wheels }\end{array}$ & 1.4 \\
\hline$l[\mathrm{~m}]$ & Wheelbase & 2.6 \\
\hline$d_{f}[\mathrm{~m}]$ & Tread of front wheel & 1.46 \\
\hline$d_{r}[\mathrm{~m}]$ & Tread of rear wheel & 1.48 \\
\hline$h_{g}[\mathrm{~m}]$ & Height of gravitational center & 0.6 \\
\hline$\rho_{f}[-]$ & Roll stiffness front-rear distribution ratio & 0.5 \\
\hline$\rho_{r}[-]$ & Roll stiffness front-rear distribution ratio & 0.5 \\
\hline$J_{p}\left[\mathrm{kgm}{ }^{2}\right]$ & Wheel inertial moment & 0.61 \\
\hline$r[\mathrm{~m}]$ & Wheel radius & 0.31 \\
\hline$g\left[\mathrm{~m} / \mathrm{s}^{2}\right]$ & Gravitational acceleration & 9.8 \\
\hline
\end{tabular}

$$
\begin{aligned}
N_{t}= & -\left(\frac{d_{f}}{2} \sin \delta+l_{f} \cos \delta\right) F_{y f l}+\left(\frac{d_{f}}{2} \sin \delta-l_{f} \cos \delta\right) F_{y f r} \\
& +l_{r}\left(F_{y r l}+F_{y r r}\right) .
\end{aligned}
$$

The slip ratio $\lambda_{i j}$ and sideslip angle $\beta_{i j}$ are expressed as follows considering that the driving force is applied when $r \omega_{i j} \geq V_{w i j}$, and the braking force is applied when $r \omega_{i j}<V_{w i j}$.

$$
\begin{aligned}
& \lambda_{i j}=\frac{r \omega_{i j}-V_{w i j}}{\max \left(r \omega_{i j}, V_{w i j}\right)}, \\
& V_{w f l}=\left(v_{x}-\frac{d_{f} \gamma}{2}\right) \cos \delta+\left(v_{y}+l_{f} \gamma\right) \sin \delta, \\
& V_{w f r}=\left(v_{x}+\frac{d_{f} \gamma}{2}\right) \cos \delta+\left(v_{y}+l_{f} \gamma\right) \sin \delta, \\
& V_{w r l}=v_{x}-\frac{d_{r} \gamma}{2}, V_{w r r}=v_{x}+\frac{d_{r} \gamma}{2}, \\
& \beta_{f l}=\tan ^{-1}\left(\frac{v_{y}+l_{f} \gamma}{v_{x}-\frac{d_{f}}{2} \gamma}\right)-\delta, \\
& \beta_{f r}=\tan ^{-1}\left(\frac{v_{y}+l_{f} \gamma}{v_{x}+\frac{d_{f}}{2} \gamma}\right)-\delta, \\
& \beta_{r l}=\tan ^{-1}\left(\frac{v_{y}-l_{r} \gamma}{v_{x}-\frac{d_{r}}{2} \gamma}\right) \text {, } \\
& \beta_{r l}=\tan ^{-1}\left(\frac{v_{y}-l_{r} \gamma}{v_{x}+\frac{d_{r}}{2} \gamma}\right) \text {. }
\end{aligned}
$$

The driving/braking force $F_{x i j}$ and the lateral force $F_{y i j}$ acting on the tires are a function of the slip ratio $\lambda_{i j}$ and the sideslip angle $\beta_{i j}$, respectively. These forces contain the saturation characteristics that change according to the condition of the road surface. In this paper, the 
Table 2 Parameters of Magic Formula tire model

\begin{tabular}{|c|c|c|c|c|c|c|c|c|c|c|c|}
\hline \multicolumn{10}{|c|}{ Front and rear tire driving/braking force $F_{x i j}(i \in\{f, r\}, j \in\{l, r\})$} \\
\hline$B_{x i j 0}$ & 5.6 & $C_{x i j 0}$ & 1.4 & $E_{x i j 0}$ & -2.98 & $b_{x i j 1}$ & 5.0 & $b_{x i j 2}$ & 0.2 \\
\hline$\kappa_{x i j}$ & 1.0 & $C_{x i j}$ & 1.0 & $S_{x i j}$ & 0 & \multicolumn{4}{|c|}{} \\
\hline \multicolumn{8}{|c|}{ Front tire lateral force $F_{y f j}(j \in\{l, r\})$} \\
\hline$B_{y f j 0}$ & 3.7 & $C_{y f j 0}$ & 1.4 & $E_{y f j 0}$ & -2.98 & $b_{y f j 1}$ & 5.0 & $b_{y f j 2}$ & 0.2 \\
\hline$b_{y f j 3}$ & 0 & $\kappa_{y f j}$ & 1.0 & $C_{y f j}$ & 1.0 & $S_{y f j}$ & 0 & & \\
\hline \multicolumn{10}{|c|}{ Rear tire lateral force $F_{y r j}(j \in\{l, r\})$} \\
\hline$B_{y r j 0}$ & 6.7 & $C_{y r j 0}$ & 1.4 & $E_{y r j 0}$ & -2.98 & $b_{y r j 1}$ & 5.0 & $b_{y r j 2}$ & 0.2 \\
\hline$b_{y r j 3}$ & 0 & $\kappa_{y r j}$ & 1.0 & $C_{y r j}$ & 1.0 & $S_{y r j}$ & 0 & & \\
\hline
\end{tabular}

Magic Formula ${ }^{(8)}$, expressed by the following equations, is used as the tire model to express these characteristics.

$$
\begin{aligned}
F_{x i j}\left(\lambda_{i j}, \beta_{i j}\right) & =D_{x i j}\left(\lambda_{i j}\right) \cos \left[C_{x i j} \tan ^{-1}\left\{B_{x i j}\left(\lambda_{i j}\right)\left(\beta_{i j}+S_{x i j}\right)\right\}\right], \\
B_{x i j}\left(\lambda_{i j}\right) & =b_{x i j 1} \cos \left\{\tan ^{-1}\left(b_{x i j 2} \lambda_{i j}\right)\right\} \kappa_{x i j}(>0), \\
D_{x i j}\left(\lambda_{i j}\right) & =\frac{F_{x i j 0}\left(\lambda_{i j}\right)}{\cos \left[C_{x i j} \tan ^{-1}\left\{B_{x i j}\left(\lambda_{i j}\right) S_{x i j}\right\}\right]}, \\
F_{x i j 0}\left(\lambda_{i j}\right) & =\mu_{i j} F_{z i j} \sin \left[C_{x i j 0} \tan ^{-1}\left\{B_{x i j 0} \lambda_{i j}-E_{x i j 0}\left(B_{x i j 0} \lambda_{i j}-\tan ^{-1}\left(B_{x i j 0} \lambda_{i j}\right)\right)\right\}\right], \\
F_{y i j}\left(\lambda_{i j}, \beta_{i j}\right) & =D_{y i j}\left(\beta_{i j}\right) \cos \left[C_{y i j} \tan ^{-1}\left\{B_{y i j}\left(\beta_{i j}\right)\left(\lambda_{i j}+S_{y i j}\right)\right\}\right], \\
B_{y i j}\left(\beta_{i j}\right) & =b_{y i j 1} \cos \left[\tan ^{-1}\left\{b_{y i j 2}\left(\beta_{i j}-b_{y i j 3}\right)\right\}\right] \kappa_{y i j}(>0), \\
D_{y i j}\left(\beta_{i j}\right) & =\frac{F_{y i j 0}\left(\beta_{i j}\right)}{\cos \left[C_{y i j} \tan ^{-1}\left\{B_{y i j}\left(\beta_{i j}\right) S_{y i j}\right\}\right]}, \\
F_{y i j 0}\left(\beta_{i j}\right) & =\mu_{i j} F_{z i j} \sin \left[C_{y i j 0} \tan ^{-1}\left\{B_{y i j 0} \beta_{i j}-E_{y i j 0}\left(B_{y i j 0} \beta_{i j}-\tan ^{-1}\left(B_{y i j 0} \beta_{i j}\right)\right)\right\}\right],
\end{aligned}
$$

where $F_{x i j 0}\left(\lambda_{i j}\right)$ and $F_{y i j 0}\left(\beta_{i j}\right)$ are the longitudinal and lateral force in the case of pure slip, $\mu_{i j}$ is the friction coefficient of the road surface, $F_{z i j}$ is the vertical load on each wheel, and $C_{k i j}$, $S_{k i j}, b_{k i j 1}, b_{k i j 2}, b_{y i j 3}, \kappa_{k i j}, B_{k i j 0}, C_{k i j 0}$, and $E_{k i j 0}(k \in\{x, y\})$ are the design parameters. These parameters of the Magic Formula tire models Eqs. (14) and (15) are listed in Table 2. By using Eqs. (14) and (15) to express the driving/braking force $F_{x i j}$ and the lateral force $F_{y i j}$; the change in driving/braking and lateral forces can be studied as shown in Figs. 3 and 4, respectively. The vertical load on each wheel $F_{z i j}$ is expressed as follows using the longitudinal and lateral accelerations $\dot{v}_{x}, \dot{v}_{y}{ }^{(9)}$.

$$
\begin{aligned}
& F_{z f l}=m_{s f} g+\frac{1}{2} \frac{l_{r}}{l} m_{s} g-\rho_{f} \dot{v}_{y} m_{s} \frac{h_{g}}{d_{f}}-\dot{v}_{x} m_{s} \frac{h_{g}}{l}, \\
& F_{z f r}=m_{s f} g+\frac{1}{2} \frac{l_{r}}{l} m_{s} g+\rho_{f} \dot{v}_{y} m_{s} \frac{h_{g}}{d_{f}}-\dot{v}_{x} m_{s} \frac{h_{g}}{l} \\
& F_{z r l}=m_{s r} g+\frac{1}{2} \frac{l_{f}}{l} m_{s} g-\rho_{r} \dot{v}_{y} m_{s} \frac{h_{g}}{d_{r}}+\dot{v}_{x} m_{s} \frac{h_{g}}{l}, \\
& F_{z r r}=m_{s r} g+\frac{1}{2} \frac{l_{f}}{l} m_{s} g+\rho_{r} \dot{v}_{y} m_{s} \frac{h_{g}}{d_{r}}+\dot{v}_{x} m_{s} \frac{h_{g}}{l} .
\end{aligned}
$$

Physical parameters $g, m_{s}, m_{s i}, \rho_{i}, l, l_{i}, d_{i}$, and $h_{g}$ are summarized in Table 1 . The vertical load $F_{z i j}$, driving/braking force $F_{x i j}$, and lateral force $F_{y i j}$ on each wheel must satisfy the following equation in all cases ${ }^{(10)}$.

$$
\mu_{i j} F_{z i j}=\sqrt{F_{x i j}^{2}+F_{y i j}^{2}} \text {. }
$$

\section{Control System}

Figure 5 shows the control system configuration. The vehicle driver can input the commanded total driving/braking force $F^{*}$ and steering angle $\delta^{*}$. The commanded yaw rate $\gamma^{*}$ is 


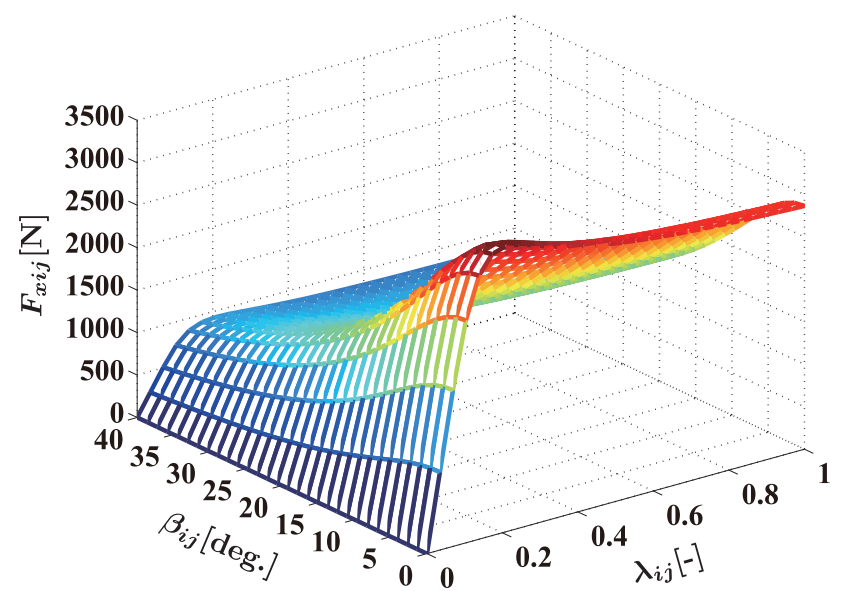

Fig. 3 Driving/braking force

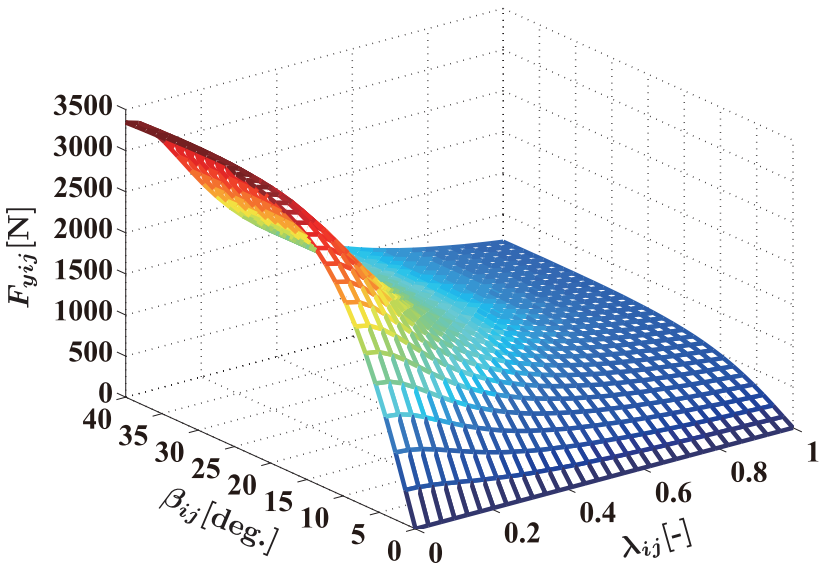

Fig. 4 Lateral force

the output of the reference model. Since the vehicle has a saturation characteristic, in practice, we employ the following reference model that approximates a saturation characteristic ${ }^{(1)}$ :

$$
\gamma^{*}(s)=\frac{1}{T_{r} s+1} \frac{g_{r} v_{x}}{\left\{1+A_{S F} v_{x}^{2}\right\} l} \frac{1}{b_{0}} \tan ^{-1}\left\{b_{0} \delta^{*}(s)\right\},
$$

where, $T_{r}[\mathrm{~s}]$ is the time constant, $g_{r}[-]$ is the steering gear ratio, $A_{S F}$ is the stability factor, and $b_{0}$ is the design parameter that defines the maximum value of $\gamma^{*}$.

The control objective is to ensure that the yaw rate $\gamma$ conforms to the commanded vehicle yaw rate $\gamma^{*} . M_{z}^{*}$ is calculated using Eq. (3) by approximate differentiation with respect to $\gamma^{*}$. Moreover, the error yaw rate feedback $K_{p} \gamma_{e}$ adds to $M_{z}^{*}$ as moment compensation in order to eliminate the yaw rate tracking error $\gamma_{e}$, where $\gamma_{e}=\gamma^{*}-\gamma$ and $K_{p}$ is the positive constant gain. By subtracting the lateral force moment $N_{t}$ from $\bar{M}_{z}^{*}$, the commanded driving/braking force differential moment $N_{z}^{*}$ is obtained. From the values of $N_{z}^{*}$ and $F^{*}$, the driving/braking force $F_{x i j}^{*}$ and control torque $\tau_{i j}^{*}$ commanded to each wheel are then calculated using the driving/braking force distribution algorithm described in the next section.

Remark 1 The control system as shown in Fig. 5 is required to measure (or estimate) the lateral force $F_{y i j}$ or the lateral force moment $N_{t}$. In Ref. (12), the estimation method of the lateral force moment $N_{t}$ using a disturbance observer is proposed. In addition, the prototype of the lateral force sensor is also developed in Ref. (13). We are assuming that the lateral force $F_{y i j}$ or the lateral force moment $N_{t}$ can be measured or estimated. 


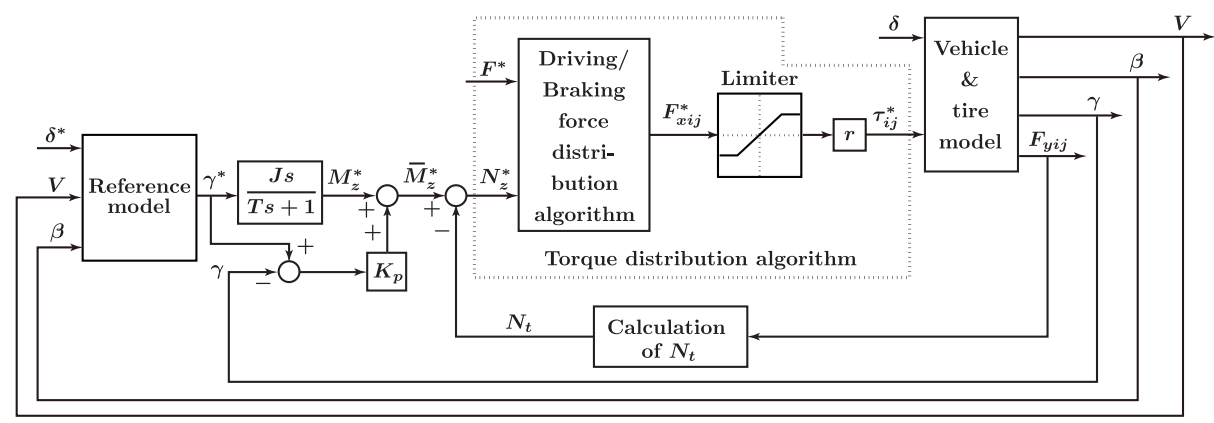

Fig. 5 Configuration of control system

\section{Driving/Braking Force Distribution Algorithm}

In order to track the yaw rate $\gamma$ to the commanded value $\gamma^{*}$, a driving/braking force must be adequately distributed to each wheel, and the required driving/braking force differential moment must be produced. Simultaneously, the constraints of vehicle control must also be satisfied. Therefore, a distribution algorithm must be applied, which has been optimized for a cost function, subject to various constraints. In this section, we describe the optimization techniques using the weighted least mean square method ${ }^{(1),(2)}$ and the quadratic programming method proposed herein to distribute the driving/braking force to the four wheels.

\subsection{Weighted least mean square method}

The driving/braking force $F^{*}$ and the driving/braking force differential moment $N_{z}^{*}$ are obtained from Eqs. (5) and (7), as follows:

$$
\begin{aligned}
& {\left[\begin{array}{c}
F^{*} \\
N_{z}^{*}
\end{array}\right]=\mathbf{A x},} \\
& \mathbf{A}=\left[\begin{array}{cccc}
\cos \delta & \cos \delta & 1 & 1 \\
l_{f} \sin \delta-\frac{d_{f}}{2} \cos \delta & \frac{d_{f}}{2} \cos \delta+l_{f} \sin \delta & -\frac{d_{r}}{2} & \frac{d_{r}}{2}
\end{array}\right],
\end{aligned}
$$

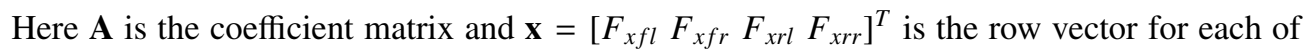
the wheel driving/braking forces. The load factor on each wheel is defined as follows:

$$
\eta_{i j}=\frac{\sqrt{F_{x i j}^{2}+F_{y i j}^{2}}}{F_{z i j}}
$$

The cost function $J$ is set, as expressed by the following equation, so as to minimize the sumof-squares for the load factor on each wheel. W in Eq. (24) is expressed by the weighted matrix in Eq. (25).

$$
\begin{aligned}
& J=\frac{F_{x f l}^{2}}{F_{z f l}^{2}}+\frac{F_{x f r}^{2}}{F_{z f r}^{2}}+\frac{F_{x r l}^{2}}{F_{z r l}^{2}}+\frac{F_{x r r}^{2}}{F_{z r r}^{2}}=\mathbf{x}^{T} \mathbf{W} \mathbf{x}, \\
& \mathbf{W}=\operatorname{diag}\left\{\frac{1}{F_{z f l}^{2}}, \frac{1}{F_{z f r}^{2}}, \frac{1}{F_{z r l}^{2}}, \frac{1}{F_{z r r}^{2}}\right\} .
\end{aligned}
$$

The weighted least mean squares solution for minimizing the cost function $J$ is expressed by the following equation:

$$
\mathbf{x}=\mathbf{W}^{-1} \mathbf{A}^{T}\left(\mathbf{A W}^{-1} \mathbf{A}^{T}\right)^{-1}\left[\begin{array}{c}
F^{*} \\
N_{z}^{*}
\end{array}\right] .
$$

Thus, a driving/braking force that minimizes and equalizes the load factor and satisfies the condition given by Eq. (22) is distributed to each wheel. 


\subsection{Quadratic programming: proposed method 1}

At the time of negotiating sharp turns and driving at high speeds, the distributed braking/driving force on each wheel, described in the previous section, may exceed the prescribed limits. The yaw rate tracking capability deteriorates at that time. Thus, some method is required to compensate for the yaw moment, for example, the used of the feedback of the yaw rate tracking error. However, the force distribution method should prevent the exceeding of the limits because a simple system with a small number of controllers is generally preferable.

In this study, quadratic programming was used in the driving/braking force distribution method; in this method, the minimum solution $\mathbf{x}$ can be found for the cost function $J$ expressed by Eq. (24), with the various constraints. Using the weighted least mean squares method, only the equality constraint can be set. On the other hand, when the the quadratic programming method is employed, it is possible to set both the equality and inequality constraints, and the bound constraint of variable $\mathbf{x}$. The object function $f(\mathbf{x})$, constraint functions $g_{1}(\mathbf{x})$ and $g_{2}(\mathbf{x})$, and the bound constraint of $\mathbf{x}$ are defined as follows:

$$
\begin{aligned}
& \min f(\mathbf{x})=J=\mathbf{x}^{T} \mathbf{W} \mathbf{x}=\frac{F_{x f l}^{2}}{F_{z f l}^{2}}+\frac{F_{x f r}^{2}}{F_{z f r}^{2}}+\frac{F_{x r l}^{2}}{F_{z r l}^{2}}+\frac{F_{x r r}^{2}}{F_{z r r}^{2}}, \\
& \text { s.t. } \quad g_{1}(\mathbf{x}):\left\{\begin{array}{ll}
\hat{F}_{x} \leq F^{*}, & \text { if } F^{*} \geq 0 \\
\hat{F}_{x}>F^{*}, & \text { if } F^{*}<0
\end{array}, g_{2}(\mathbf{x}):\left\{\begin{array}{ll}
\hat{N}_{z} \geq N_{z}^{*}, & \text { if } N_{z}^{*} \geq 0 \\
\hat{N}_{z}<N_{z}^{*}, & \text { if } N_{z}^{*}<0
\end{array}\right. \text {, }\right. \\
& \hat{F}_{x}=\left(F_{x f l}^{*}+F_{x f r}^{*}\right) \cos \delta+F_{x r l}^{*}+F_{x r r}^{*}, \\
& \hat{N}_{z}=\left(l_{f} \sin \delta-\frac{d_{f}}{2} \cos \delta\right) F_{x f l}^{*}+\left(\frac{d_{f}}{2} \cos \delta+l_{f} \sin \delta\right) F_{x f r}^{*} \\
& -\frac{d_{r}}{2} F_{x r l}^{*}+\frac{d_{r}}{2} F_{x r r}^{*}
\end{aligned}
$$

where, $\mathbf{F}_{\text {llim }}$ and $\mathbf{F}_{\text {ulim }}$ are the lower and upper limit values, respectively, for the driving/braking force. The inequality constraint $g_{1}(\mathbf{x})$ implies that the commanded driving/braking force $F_{x i j}^{*}$ for each wheel must be less than or equal to the total commanded driving/braking force $\left|F_{x}^{*}\right|$, and $g_{2}(\mathbf{x})$ means that the driving/braking force differential moment produced by driving/braking forces $F_{x i j}^{*}$ on each wheel must be greater than or equal to the commanded value of $\left|N_{z}^{*}\right|$. Furthermore, to obtain a large yaw moment, the inequality constraint is used instead of the equality constraint.

\subsection{Control method based on moment compensation: proposed method 2}

Even for the methods described in the previous section, the driving/braking force of each wheel will exceed the limits in some cases. This not only deteriorates the yaw rate tracking performance during the saturation of the driving/braking force of each wheel, but it deteriorates also immediately after resolving the saturation of the driving/braking force of each wheel. In order to prevent the yaw rate tracking performance immediately after resolving the saturation of the driving/braking force of wheel, a moment compensator that feeds back the moment $\bar{N}_{z}$ difference between the limit value and the commanded driving/braking forces $F_{x i j}^{*}$ and increases the driving/braking force differential moment $N_{z}$ is added to the control system, as shown in Fig. 6. The delay block in Fig. 6 is used to delay the feedback of $\bar{N}_{z}$. When the driving/braking forces distributed by quadratic programming exceed the limits, using this type of compensator in the system proves to be the most efficient control method.

Remark 2 Design parameter $T_{d}$ of the delay block in Fig. 6 is the parameter to set the time to add $\bar{N}_{z}$. By setting $T_{d}$ to a very small value, the yaw rate tracking performance is rarely different from proposed method 1 because $\bar{N}_{z}$ is added during $N_{z}$ is saturated. In contrast, By setting $T_{d}$ to a very large value, the yaw rate tracking performance is worse than proposed 


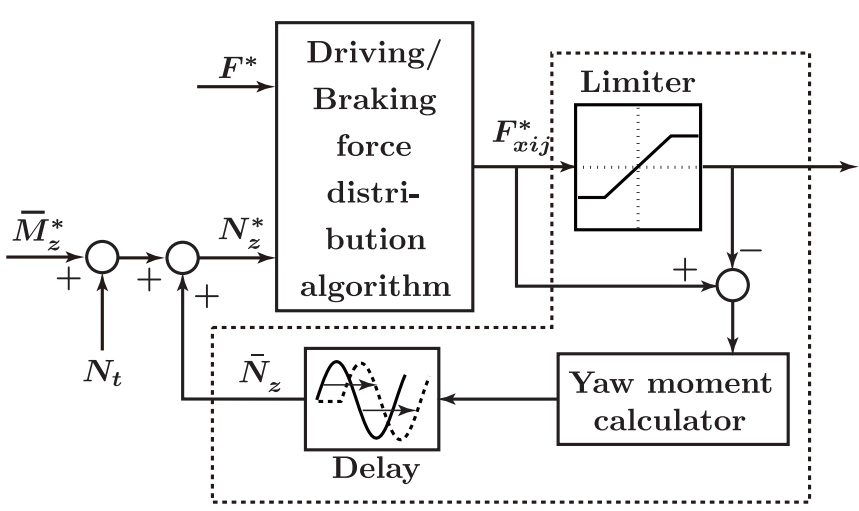

Fig. 6 Control system with yaw moment compensator.

Table 3 Simulation conditions

\begin{tabular}{|c|c|}
\hline$\delta^{*}:$ Fig. 7 & Command of steering angle \\
\hline$F^{*}:$ Fig. 8 & Command of driving/braking force \\
\hline$v_{x} 0=25, v_{y}(0)=0[\mathrm{~m} / \mathrm{s}]$ & Initial velocity \\
\hline$\gamma(0)=0[\mathrm{rad} / \mathrm{s}]$ & Initial yaw rate \\
\hline$\omega_{i j}(0)=81[\mathrm{rad} / \mathrm{s}]$ & Initial angular velocity of each wheel \\
\hline $\mathbf{F}_{\text {Ilim }} / \mathbf{F}_{\text {ulim }}:-350 / 350[\mathrm{~N}]$ & Saturation value of the limiter \\
\hline$g_{r}=1 / 10[-]$ & Steering gear ratio \\
\hline$\mu_{i j}=0.9[-]$ & Road frictional coefficient \\
\hline$K_{p}=2000$ & Positive constant gain \\
\hline$b_{0}=2$ & Design parameter of reference model $(21)$ \\
\hline$T_{r}=0.1[\mathrm{~s}]$ & Time constant of reference model $(21)$ \\
\hline$T=0.01[\mathrm{~s}]$ & Time constant of approximation differentiator \\
\hline$T_{d}=0.4[\mathrm{~s}]$ & Delay time of delay block \\
\hline
\end{tabular}

method 1 because the saturation of the driving/braking force of each wheel is resolved, and after a while, $\bar{N}_{z}$ is added. In numerical simulation at $\S 5, T_{d}$ is set to a value of 0.4 by trial and error.

\section{Simulation and Method Comparison}

Table 3 shows the simulation conditions. The lower limit value $\mathbf{F}_{\text {llim }}$ and upper limit value $\mathbf{F}_{\text {ulim }}$ are determined by the maximum torque of the in-wheel motor of a commercial electric vehicle. The present simulation deals with cases of high-speed driving where (a) comparatively mild steering is applied, and (b) hard steering is applied. The driving/braking force distribution methods in the simulation include the methods using the weighted least mean squares (conventional method), using quadratic programming (proposed method 1), and using a moment compensator along with quadratic programming (proposed method 2). In the proposed methods 1 and 2, the active set method is utilized as a technique for solving convex quadratic programming problems. Moreover, in order to avoid that scenario where the driving/braking force differential moment $N_{z}^{*}$ becomes zero, the following object function, which includes the linear term $\mathbf{c}^{T} \mathbf{x}$ is used:

$$
f(\mathbf{x})=\mathbf{x}^{T} \mathbf{W} \mathbf{x}+\mathbf{c}^{T} \mathbf{x}, \mathbf{c}=-\left[\begin{array}{llll}
0.01 & 0.01 & 0.01 & 0.01
\end{array}\right]^{T} .
$$

Figures 9 and 10 show the simulation results of case (a), and Fig. 11 shows the vehicle trajectory and the lateral acceleration. When the conventional method is used, the limits are exceeded when the driving/braking force is distributed during steering angle changes. However, when using proposed method 1 , the bound constraints set by quadratic programming proved effective, and the achieved driving/braking force distribution was well within the limits. In this manner, the required driving/braking force differential moment is produced, the commanded yaw rate is achieved without any delays, and the swell of vehicle trajectory is suppressed. In addition, in order to verify the effectiveness of constraint $g_{2}(\mathbf{x})$, the simulation when applied 


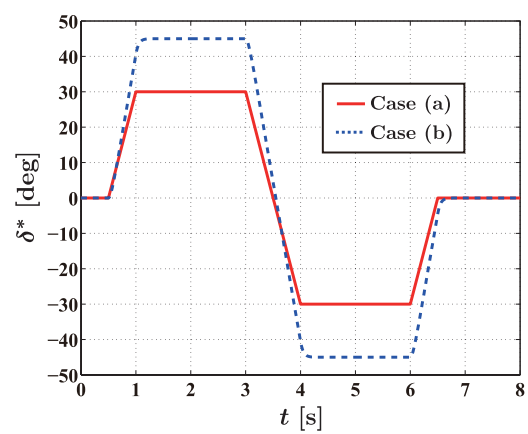

Fig. 7 Command of steering angle $\delta^{*}$

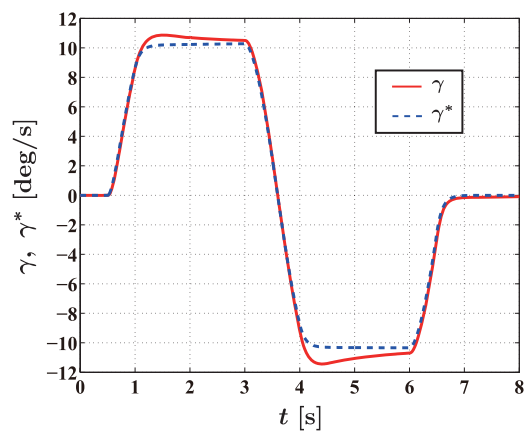

(i) $\gamma$

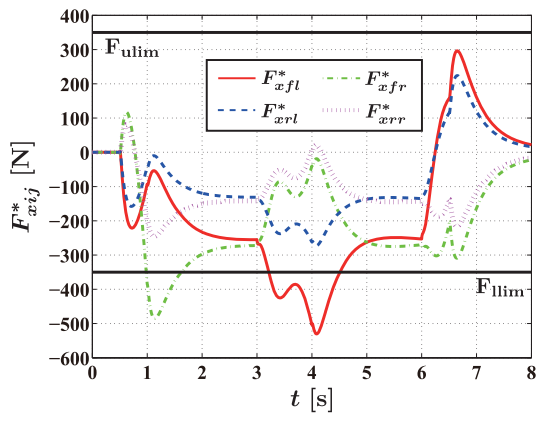

(iii) $F_{x i j}^{*}$

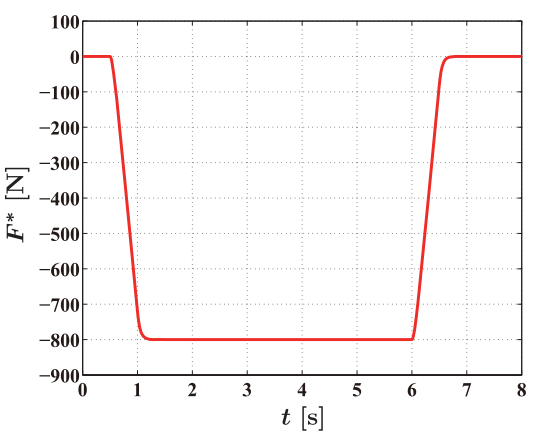

Fig. 8 Command of driving/braking force $F^{*}$

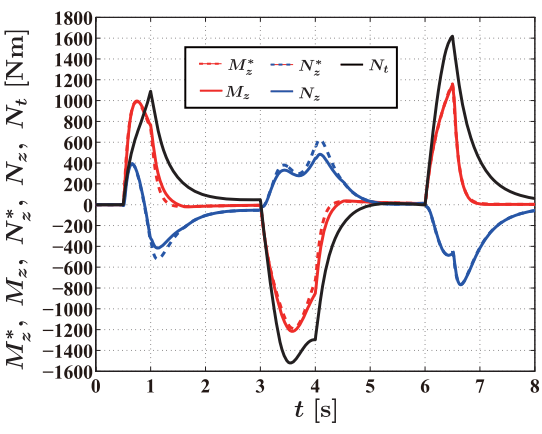

(ii) $M_{z}^{*}, M_{z}, N_{z}^{*}, N_{z}$ and $N_{t}$

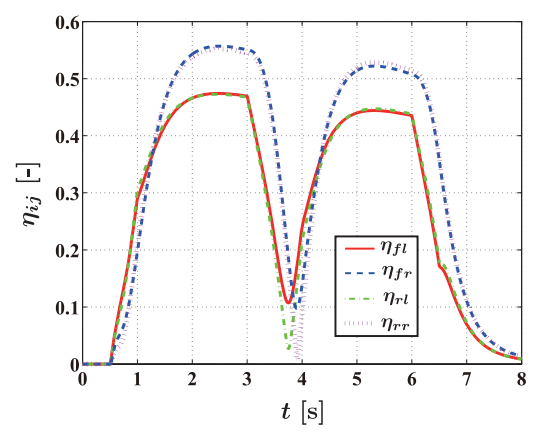

(iv) $\eta_{i j}$

Fig. 9 Simulation results of conventional method (case (a))

the proposed method 1 without the constraint $g_{2}(\mathbf{x})$ was performed. Figure 12 shows this simulation results. In Fig. 12, although the driving/braking force was well within the limits, yaw rate tracking performance deteriorate drastically because sufficient driving/braking differential moment for turn of vehicles was not generated. From this result, it turns out that it can prevent falling into this situation by adding the constraint $g_{2}(\mathbf{x})$.

Figures 13, 14, and 15 show the simulation results of case (b). When the conventional method is used, the driving/braking force exceeds the limits considerably, and the error between the yaw moment $M_{z}$ and the commanded yaw moment $M_{z}^{*}$ becomes large. Consequently, a large delay occurrs in achieving the commanded yaw rate on completion of the steering angle change. Even though moment compensation is provided as error yaw rate feedback, $K_{p} \gamma_{e}$ does cause an increase in the $N_{z}^{*}$ value, and the yaw rate tracking performance obviously deteriorates as seen in Fig. 13(i).

On the other hand, even for the proposed method 1 , the limits are exceeded during steering angle changes. This result could be attributed to the fact that in order to generate the driving/braking force differential moment $N_{z}$ that satisfies the inequality constraint $g_{2}(\mathbf{x})$, the commanded driving/braking force $F_{x i j}^{*}$ needs to be greater than the limit because the commanded driving/braking force differential moment $N_{z}^{*}$ is large when compared to case (a). However, because the proposed method takes into account the limit of the driving/braking 


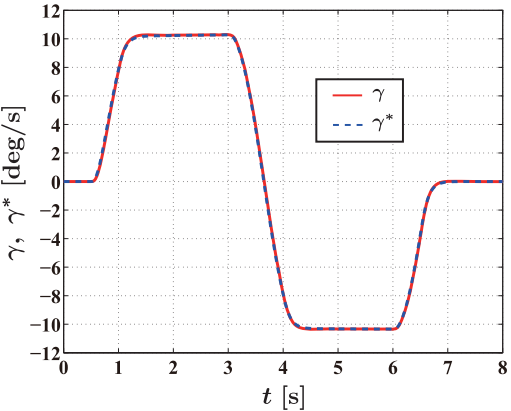

(i) $\gamma$

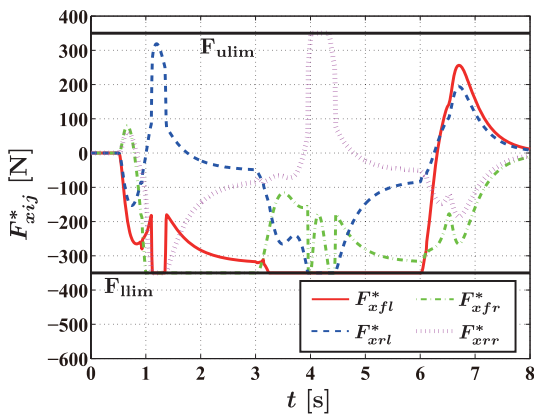

(iii) $F_{x i j}^{*}$

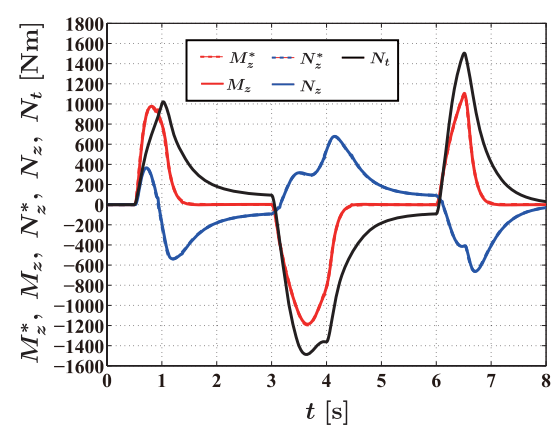

(ii) $M_{z}^{*}, M_{z}, N_{z}^{*}, N_{z}$ and $N_{t}$

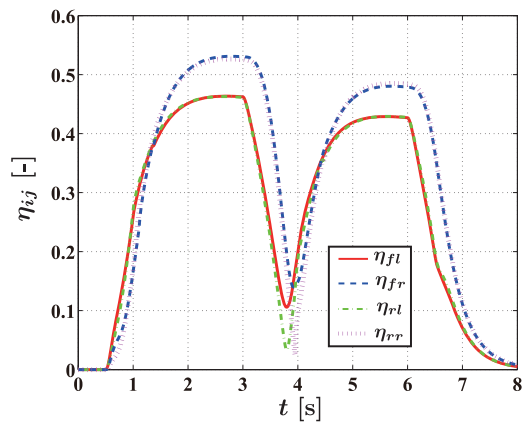

(iv) $\eta_{i j}$

Fig. 10 Simulation results of proposed method 1 (case (a))

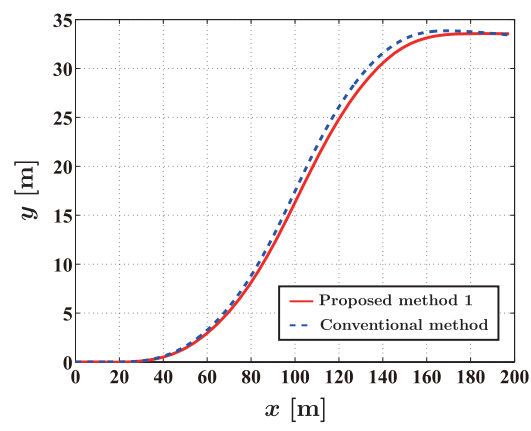

(i) Vehicle trajectory

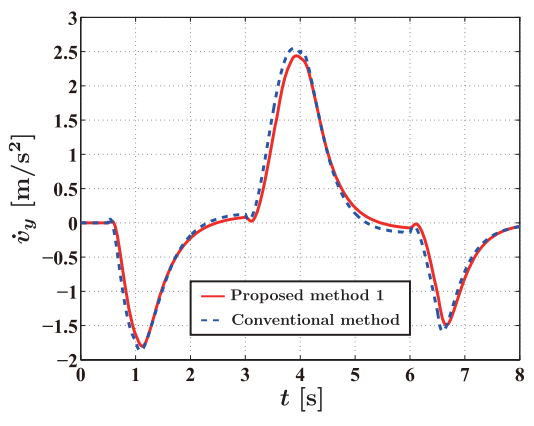

(ii) $\dot{v}_{y}$

Fig. 11 Comparison of vehicle trajectory and lateral acceleration (case (a))

force, the error between the yaw moment $M_{z}$ and the commanded yaw moment $M_{z}^{*}$ becomes small in comparison to the conventional method. Consequently, the yaw rate tracking error also becomes small. Moreover, the proposed method 2 has the yaw moment compensator, and in this method, the distributed driving/braking force produces a large moment after the steering angle change because the moment $\bar{N}_{z}$ difference between the limit value and the commanded driving/braking forces $F_{x i j}^{*}$ are added after the delay of $T_{d}$ seconds. The proposed method 2, therefore, exhibited an even faster convergence to the yaw rate command value. 


\section{Dynamics}

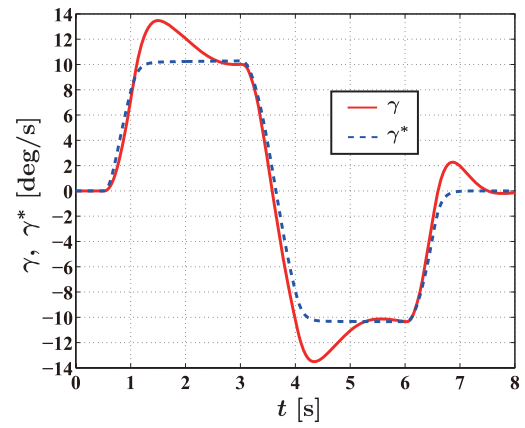

(i) $\gamma$

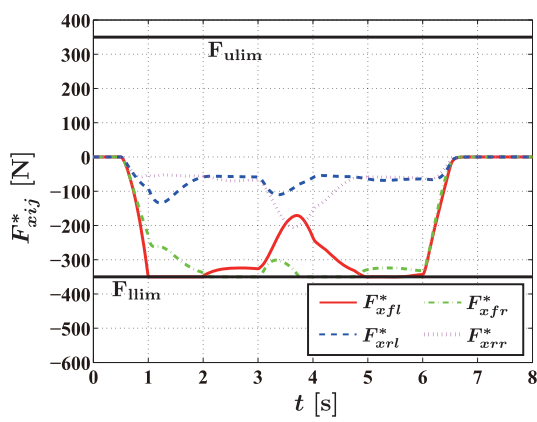

(iii) $F_{x i j}^{*}$

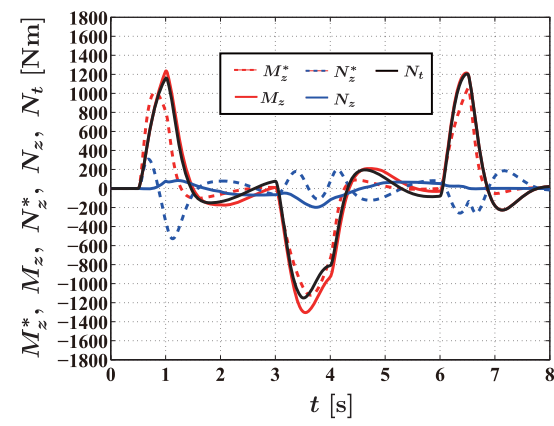

(ii) $M_{z}^{*}, M_{z}, N_{z}^{*}, N_{z}$ and $N_{t}$

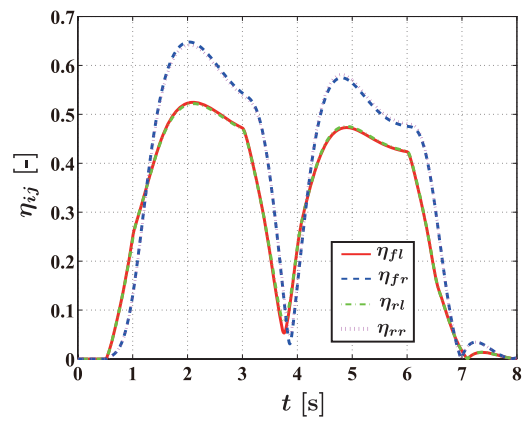

(iv) $\eta_{i j}$

Fig. 12 Simulation results of proposed method 1 without inequality constraint $g_{2}(\mathbf{x})$ (case (a))

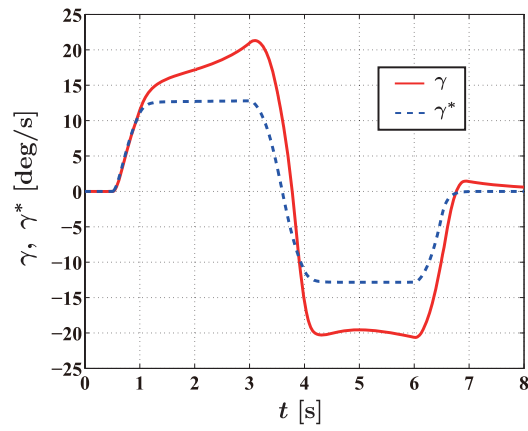

(i) $\gamma$

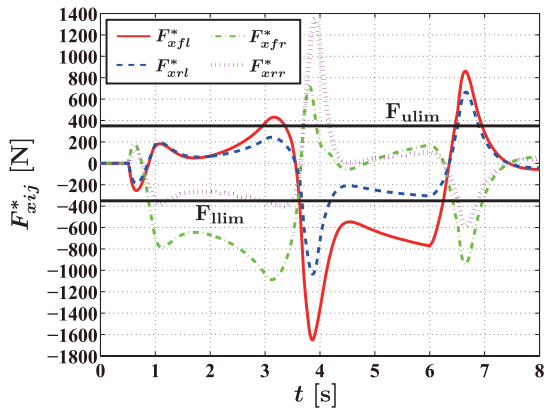

(iii) $F_{x i j}^{*}$

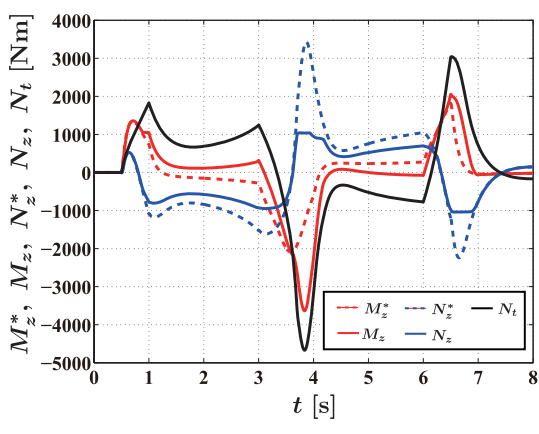

(ii) $M_{z}^{*}, M_{z}, N_{z}^{*}, N_{z}$ and $N_{t}$

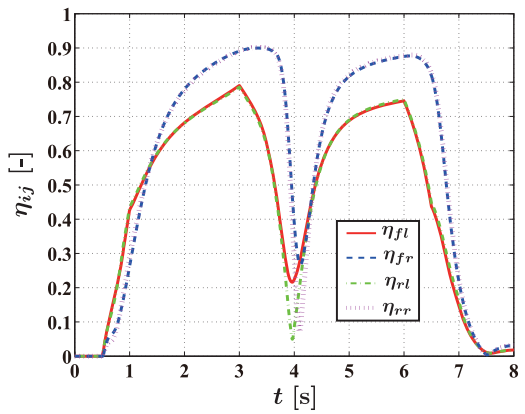

(iv) $\eta_{i j}$

Fig. 13 Simulation results of conventional method (case (b)) 


\section{Dynamics}

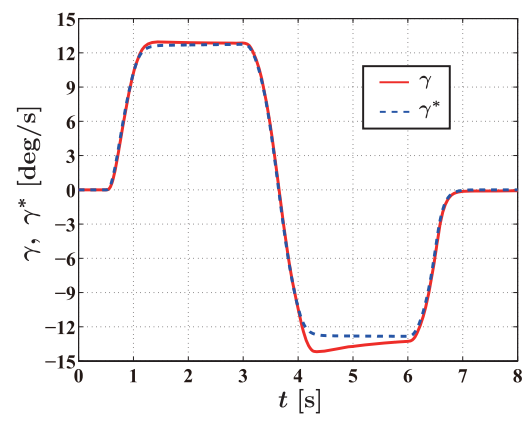

(i) $\gamma$

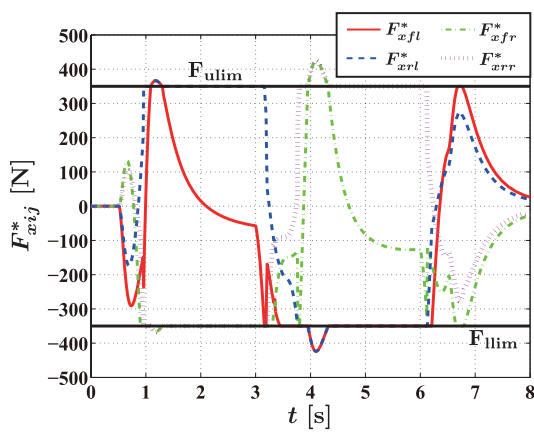

(iii) $F_{x i j}^{*}$

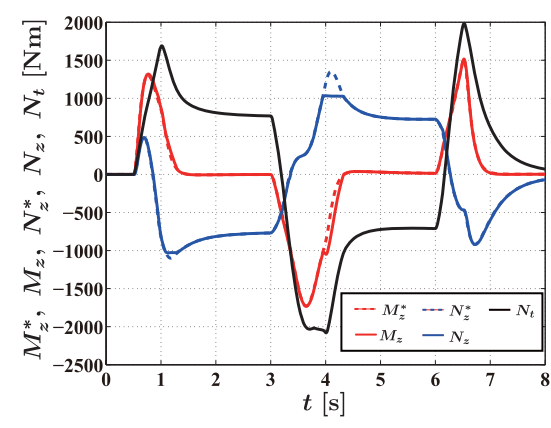

(ii) $M_{z}^{*}, M_{z}, N_{z}^{*}, N_{z}$ and $N_{t}$

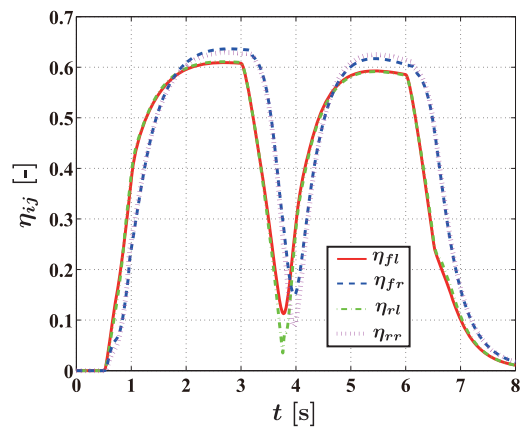

(iv) $\eta_{i j}$

Fig. 14 Simulation results of proposed method 1 (case (b))

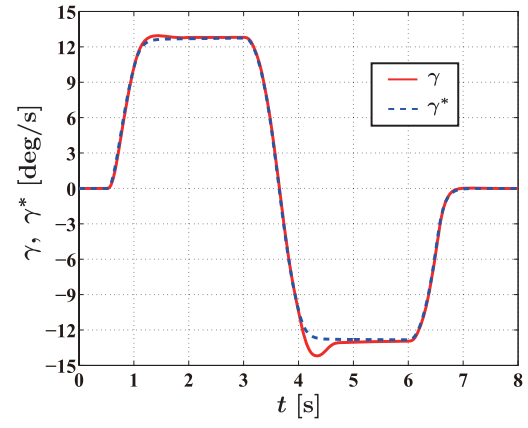

(i) $\gamma$

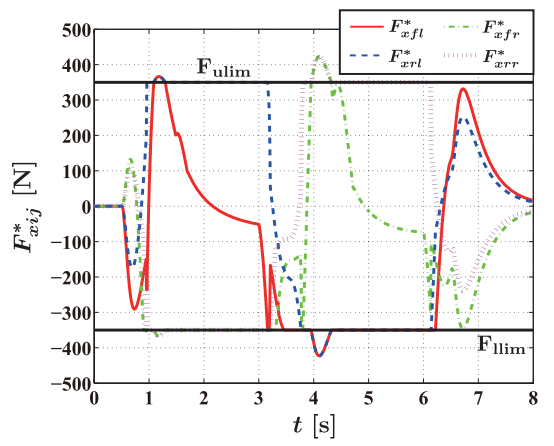

(iii) $F_{x i j}^{*}$

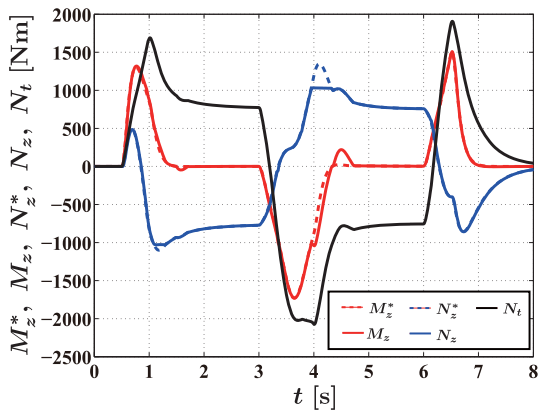

(ii) $M_{z}^{*}, M_{z}, N_{z}^{*}, N_{z}$ and $N_{t}$

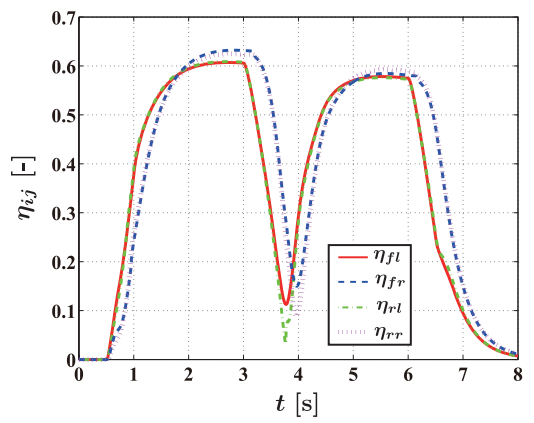

(iv) $\eta_{i j}$

Fig. 15 Simulation results of proposed method 2 (case (b)) 


\section{Conclusions and Future Works}

In this paper, we propose a yaw rate tracking control method for distributing the vehicle driving/braking force at the time of negotiating sharp turns and driving at high speeds. This method employs quadratic programming, which distributes the driving/braking force on all wheels in order to equalize the tire load factor and limit the driving/braking force. The yaw rate tracking performance can be improved even while negotiating sharp turns and driving at high speeds by imposing appropriate limits, for example, on the driving/braking force differential moment. A numerical simulation verifies the validity of our proposed method.

A torque (maximum torque) larger than the rated torque can be produced during transient operations of the actual motor. Utilizing this large torque to improve the control system is a subject for future studies.

\section{References}

(1) Ando, N., and Fujimoto, H., Yaw-rate Control for Electric Vehicle with Active Front/Rear Steering and Driving/Braking For Distribution of Rear Wheels, IEE of Japan Technical Meeting Record, pp.41-46, 2009 (in Japanese)

( 2 ) Ando, N., and Fujimoto, H., Fundamental Study of Intergrated Control for Active Front/Rear Steering and Driving/Braking For Distribution Based on Least Squares Solution for Electric Vehicle, IEE of Japan Technical Meeting Record, pp.35-40, 2008 (in Japanese)

( 3 ) Sakai, S., Sado, H., and Hori, Y., Dynamic driving/braking force distribution in electric vehicles with independently driven four wheels, Electrical Engineering in Japan, Vol.138, No.1, pp.79-89, 2002

( 4 ) Hiraoka, T., Nishihara, O., and Kumamoto, H., Driving/Braking Force Distribution by Minimax Optimization of Tire Workload (Case of Active Four-Wheel Steering for ZeroSideslip Control), Transactions of the Japan Society of Mechanical Engineers, Series C, Vol.71, No.704, pp.1238-1246, 2005 (in Japanese)

( 5 ) Fredriksson, J., Andreasson, J., and Laine, L., Wheel Force Distribution for Improved Handling in a Hybrid Electric Vehicle using Nonlinear Control, 43rd IEEE Conference on Decision and Control, pp.4081-4086, 2004

( 6 ) Plumlee, J.H., Bevly, D.M., and Hodel, A.S., Control of A Ground Vehicle Using Quadratic Programming Based Control Allocation Techniques, Proceedings of the 2004 American Control Conference, pp.4704-4709, 2004

( 7 ) Tondel, P., and Johansen, T.A., Control Allocation for Yaw Stabilization in Automotive Vehicles using Multiparametric Nonlinear Programming, Proceedings of the 2005 American Control Conference, pp.453-458, 2005

( 8 ) Pacejka, H.B., and Besselink, I.J., Magic Formula Tyre Model with Transient Properties, Vehicle System Dynamics Supplement, Vol.27, pp.234-249, 1997

( 9 ) Huh, K., Seo, C., Kim, J., and Hong, D., Active Steering Control Based on the Estimated Tire Forces, Proceedings of the 1999 American Control Conference, pp.729-733, 1999

(10) Ono, E., Hattori, Y., and Muragishi, Y., Estimation of Tire Friction circle and Vehicle Dynamics Integrated Control for Four-wheel Distributed Steering and Four-wheel Distributed Traction/Braking Systems, REDD Review of Toyota CRDL, Vol.40, No.4, pp.713,2005

(11) Fujii, N., and Horiuchi, S., Nonlinear Integrated Control of Vehicles using Backstepping Method, The 15th Transportation and Logistics 2011 (TRANSLOG2011), pp.257-260, 2006 (in Japanese)

(12) Ando, N., and Fujimoto, H., Yaw-rate Control for Electric Vehicle with Active Front/Rear Steering and Driving/Braking Force Distribution of Rear Wheels, The 11th IEEE International Workshop on Advanced Motion Control, pp.726-731, 2010

(13) NSK Ltd. press release: URL: http://www.jp.nsk.com/company/presslounge/news/2005/ press05101801.html (in Japanese). 